\title{
OPTICAL DISPERSION RELATIONS FOR "DIAMONDLIKE" CARBON FILMS
}

Samuel A. Alterovitz", Robert M. Siegt, Neil S. Shoemaker $\neq$, and

John J. Pouch*

* NASA Lewis Research Center, Cleveland, Ohio 44135

tUndergraduate Student Intern at NASA Lewis Research Center from Cleveland, State University, Dept. of Electrical Engineering. Cleveland, Ohio 44106

Fundergraduate Student Intern at NASA Lewis Research Center from Case Western Reserve University, Physics Dept., Cleveland, Ohio 44115

\section{ABSTRACT}

Ellipsometric measurements on plasma deposited "diamondlike" amorphous carbon $(\mathrm{a}-\mathrm{C}: \mathrm{H})$ films were taken in the visible, ( $E=1.75$ to $3.5 \mathrm{eV})$. The films were deposited on $S i$ and their properties were varied using high temperature (up to $750^{\circ} \mathrm{C}$ ) anneals. The real $(n)$ and imaginary (k) parts of the complex index of refraction $N$ were obtained simultaneously. Following the theory of Forouhi and Bloomer (Phys. Rev. B34, 7018 (1986)), a least squares fit was used to find the dispersion relations $n(E)$ and $k(E)$. Reasonably good fits were obtained, showing that the theory can be used for a-C:H films. Morever, the value of the energy gap Eg obtained in this way was compared to the Eg. value using conventional Tauc plots and reasonably good agreement was obtained.

\section{INTRODUCTION}

The optical energy band gap of amorphous materials is usually found using a Tauc plot [1], i.e.. an extrapolation of (anE) $/ / 2$ versus $E$. Here $\alpha$ is the optical absorption coefficient, $n$ is the refractive index and $E$ is the energy. In many cases, the refractive index is almost constant or is unavailabie, and ( $\alpha E$ ) $1 / 2$ versus $E$ is used (simplified Tauc). There are several drawbacks to the Tauc theory and procedure. First, the theory relates to absorption only, and cannot give the refractive index through the Kramers-Kronig relation [2]. Second, there is ambiguity on the energy range that the Tauc extrapolation is correct. Below a certain value of $\alpha$, the absorption falls exponentially. This regime is called the Urbach edge [1]. The onset for this regime varies and values of order $\alpha=10^{3} \mathrm{~cm}^{-1}[1]$ up to $\alpha=10^{4} \mathrm{~cm}^{-1}$ [3] have been used. Empirically, many Tauc plots also start to deviate from a straight line at high $\alpha$ values $[4,5]$.

Recently, an extension of the theory of optical absorption has been published for amorphous materials [2]. Later, the theory was extended to include more than one critical point. and was applied to crystalline semiconductors [6]. In the Tauc plot derivation [1], the main assumptions are a constant matrix element and parabolic density of states for both the valence and the conduction bands. However, if the excited state has a finite lifetime $\tau$, the absorption probability has a damping factor [2] and Tauc plots are theoretically incorrect. The extinction coefficient $k$ $(k=\alpha c h / 2 E$ where $E$ is the energy) is calculated in [2,6] using the iffetime concept, obtaining.

$$
k(E)=\frac{A\left(E-E g^{2}\right.}{E^{2}-B E+C}
$$

Here $A$ is $K(\infty)$ and is proportional to $M^{2} / \tau$ where $M$ is the position matrix element $(M=\langle f|x| i\rangle) ; B=2\left(E_{c, c r i t}-E_{v, c r i t}\right)$, where $E_{c, c r i t}$ and $E_{v, c r i t}$ are energies in the conduction and valence band respectively corresponding to a critical point, i.e.. where $k\left(E_{c}, c r i t-E_{v}, c r i t\right)$ is a maximum. $C$ is related to the ijfetime $\tau$ through $h / \tau=\left(4 C^{C r}-B^{2}\right) 1 / 2$ and

Mat. Res. Soc. Symp. Proc. Vol. 152. 01989 Materials Research Society 
$E g=E_{c \text {, bottom }}-E_{v}$,top, i.e., the optical bandgap. Ising the KramersKronig relation, the refractive index was obtained [2]:

$$
n(k)=n(\infty)+\frac{B_{0} E+C_{0}}{E^{2}-B E+C}
$$

Here $B_{O}$ and $C_{0}$ are related to $A, B$ and $C$ through simple algebraic formulas, and $n(\infty)$ is a constant.

In this paper we will examine if this new result applies to "diamondlike" carbon, al so denoted a-C:H (amorphous hydrogenated carbon). If applicable, the result can give the $n(\varepsilon)$ function from a known absorption spectrum $k(E)$ and a single refractive index measurement. Also, the functions $n(E)$ and/or $k(E)$ can be used when their analytical form is required, e.g., optimizing antireflection filters. In addition, this paper will discuss the meaning of the experimental constants, A,B, C, and Eg.

Amorphous hydrogenated carbon material is made almost exclusively in form of thin films. The natural choice for an experimental technique is ellipsometry. The multiple angle of incidence, multiple wavelengths (MAW) technique [ 7 to 9 ] was used. MAW gives $n$ and $k$ of the film simultaneously at all wavelengths measured. without the use of either a known dispersion relation or application of the Kramers-Kronig analysis. This technique was commonly used to analyze various semiconductor multilayer structures $[10,11]$ when $n$ and $k$ of the constituents were known. Here, the MAW technique is used in a spectroscopic way, to measure unknown $n(E)$ and $k(\varepsilon)$. The smallest value of $k$ that is accurately determined by ellipsometry is of order 0.005 , corresponding to $\alpha \cong 1000 \mathrm{~cm}^{-1}$ in the visible. Thus, the Urbach edge regime is almost eliminated from this work. Reflection ellipsometry has the added benefit of measuring the thin film samples on any substrate. In contrast to transmission experiments. As the optical energy bandgap Eg depends on the substrate material [5], possibly due to differences in conductivity, this advantage of ellipsometry can be crucial for actual applications.

\section{EXPERIMENTAL}

Samples were prepared on $3 \mathrm{in}$. diameter $5 \mathrm{i}$ substrates using a $30 \mathrm{kHz}$ plasma deposition unit. The power. $P$ used was in the range 50 to $200 \mathrm{~W}$. with a constant flow rate of $7 \times 10^{-5} \mathrm{~m}^{3} / \mathrm{min}$. Details of the growth chamber are given in [12]. Several samples cut from the $150 \mathrm{~W}$ wafer were annealed in nitrogen for $10 \mathrm{sec}$ using a rapid thermal anneal module [5]. The rotating analyzer ellipsometer set-up [13] is essentially similar to the one described in [10]. At each angle of incidence, data was taken in the wavelength range 3500 to $7300 \AA$, with $100 \AA$ intervals. Five angles of incidence were used, usually in the range $55^{\circ}$ to $75^{\circ}$. Each measurement conslsted of 100 rev, with 72 points per rev, taken at à rate of -50 rev/sec. Background subtraction was done at each point. Calibration of the absolute value of the ellipsometric parameters $\psi$ and $\Delta$ was done using [14]. The double grating monchromator was controlled by a IBM-AT personal computer that was also used for all data acquistion and analysis. $\Psi$ and $\Delta$ were obtained by Fourier analysis. The MAW inversion process was done by minimizing experimental $\psi$ and $\Delta$ (or $\tan \psi$ and $\cos \Delta$ ) versus their calculated values [9]. The model used included only the substrate and a homogeneous film. This is a reasonable approximation, as the interface of $\mathrm{a}-\mathrm{C}: \mathrm{H}$ on $\mathrm{S} i$ includes only the native oxtde and $\sim \mathrm{A} \mathrm{SiC}_{\mathrm{x}}[15]$, and our films were above 1000 \& thick.

\section{RESULTS}

Seven samples were measured. Simplified and regular Tauc plots were done for all samples. A representative olot (50 $\mathrm{W}$ sample) is given in 
Fig. I, showing a slightly concave result versus the theory. As the annealing temperature andlor deposition power rises, the experimental iauc function first becomes a straight line and later turns convex. We kept the extrapolation range to the linear part of the experimental function. A summary of the energy bandgaps obtained from regular and simplified Tauc plots, together with sample thicknesses $t$, are given in Table I. Results of $n(E)$ and $k(E)$ for the 50 and $150 \mathrm{~W}, 600^{\circ} \mathrm{C}$ samples are given in $\mathrm{Figs} 2$ and 3 respectively. Figure 2 shows a rising $n$ versus $E$ while Fig. 3 show a decreasing $n(E)$. All other samples have an interim type of behavior. Grid least square fits to Eq. (1) were done to obtain A,B,C and Eg simultaneously. With these 4 parameters fixed, the value of $n(\infty)$ was set to get the best $n(E)$ fits. The solid lines in Figs. 2 and 3 were calculated using these parameters. Results for all samples are given in Table II, while Eg is also displayed in Table I.

TABLE I a-C:H OPTICAL ENERGY GAPS IN EV

\begin{tabular}{|c|c|c|c|c|c|}
\hline$P, W$ & $T,{ }^{\circ} \mathrm{C}$ & $t, A$ & $\operatorname{Sq} r t(\alpha E)$ & $\operatorname{Sqr} t(\alpha E n)$ & Grid \\
\hline $\begin{array}{r}50 \\
100 \\
150 \\
200 \\
150 \\
150 \\
150\end{array}$ & $\begin{array}{c}- \\
- \\
- \\
- \\
400 \\
600 \\
750\end{array}$ & $\begin{array}{r}1490 \\
2305 \\
2810 \\
2960 \\
2560 \\
1320 \\
950\end{array}$ & $\begin{array}{l}1.90 \\
2.55 \\
2.36 \\
2.02 \\
2.05 \\
1.08 \\
0.57\end{array}$ & $\begin{array}{l}1.90 \\
2.35 \\
2.36 \\
2.02 \\
2.06 \\
0.89 \\
0.39\end{array}$ & $\begin{array}{l}1.64 \\
2.70 \\
2.23 \\
1.65 \\
1.90 \\
1.03 \\
0.39\end{array}$ \\
\hline
\end{tabular}

TAELE II DISPERSION RELATION PARAMETERS FOR a-C:H FILMS

\begin{tabular}{|r|c|c|c|c|c|c|}
\hline$P, W$ & $T, C$ & $A$ & $B$, eV & $C,(e V)^{2}$ & $E g, e V$ & $n(\infty)$ \\
\hline 50 & - & .06 & 6.39 & 12.67 & 1.64 & 1.63 \\
100 & - & .01 & 6.46 & 10.51 & 2.70 & 1.70 \\
150 & - & .05 & 5.90 & 9.75 & 2.23 & 1.67 \\
200 & - & .11 & 5.99 & 11.73 & 1.65 & 1.69 \\
150 & 400 & .06 & 5.10 & 7.20 & 1.90 & 1.70 \\
150 & 600 & .40 & 3.66 & 4.22 & 1.03 & 1.58 \\
150 & 750 & .85 & 1.21 & 1.85 & 0.39 & 1.71 \\
\hline
\end{tabular}




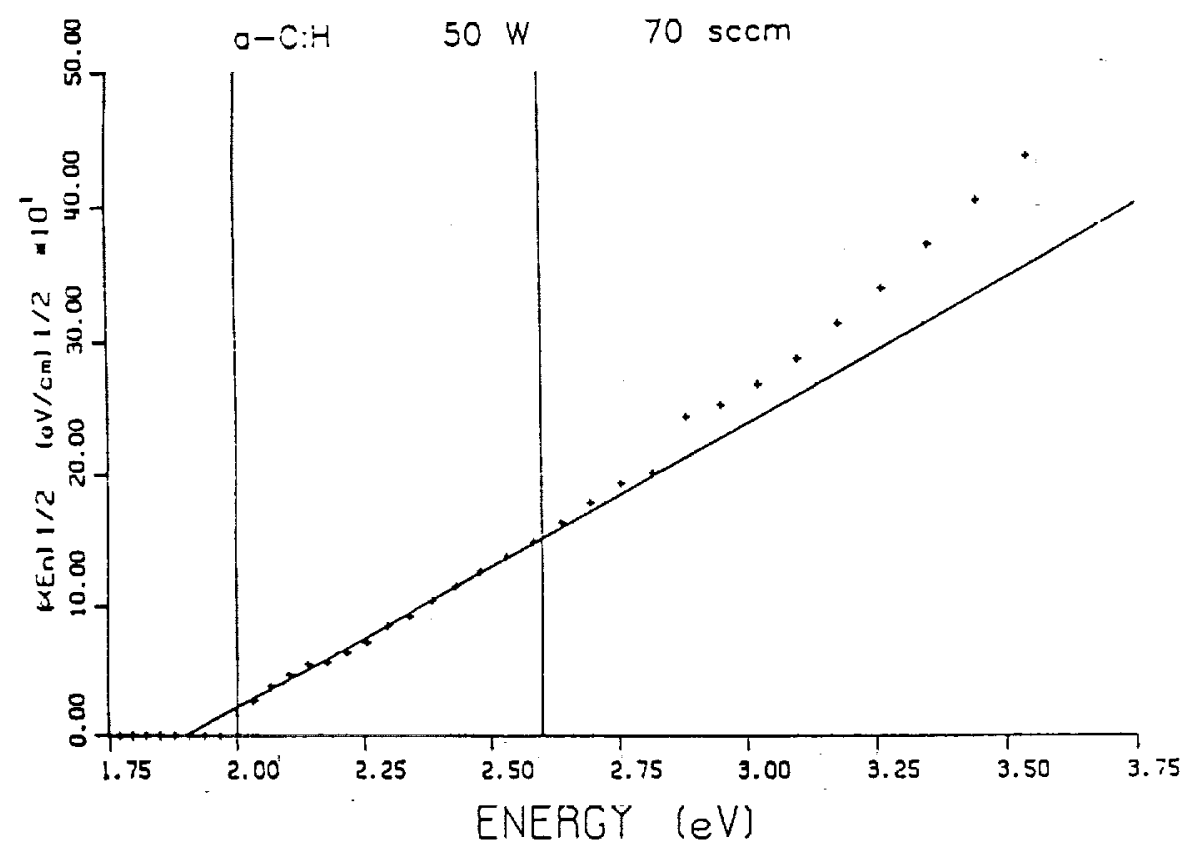

FIGURE 1. TAUC PLOT FOR THE $50 \mathrm{~W}$ SAMPLE. VERTICAL LINES SHOW THE FITTING RANGE.

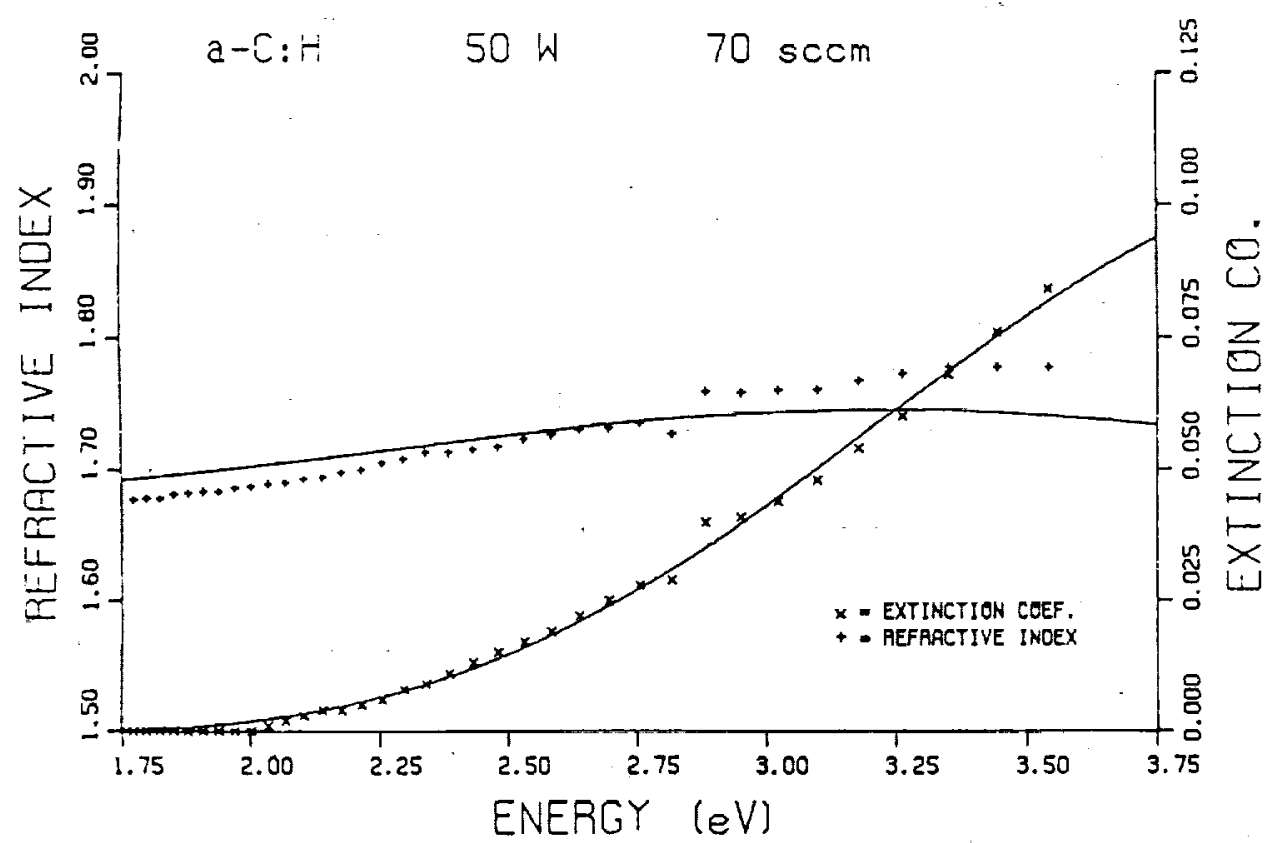

FIGURE 2. REFRACTIVE INDEX AND EXTINCTION COEFFICIENT OF THE 50 W SAMPLE. SOLID LINES ARE DUE TO EQUATIONS (1) AND (2). 


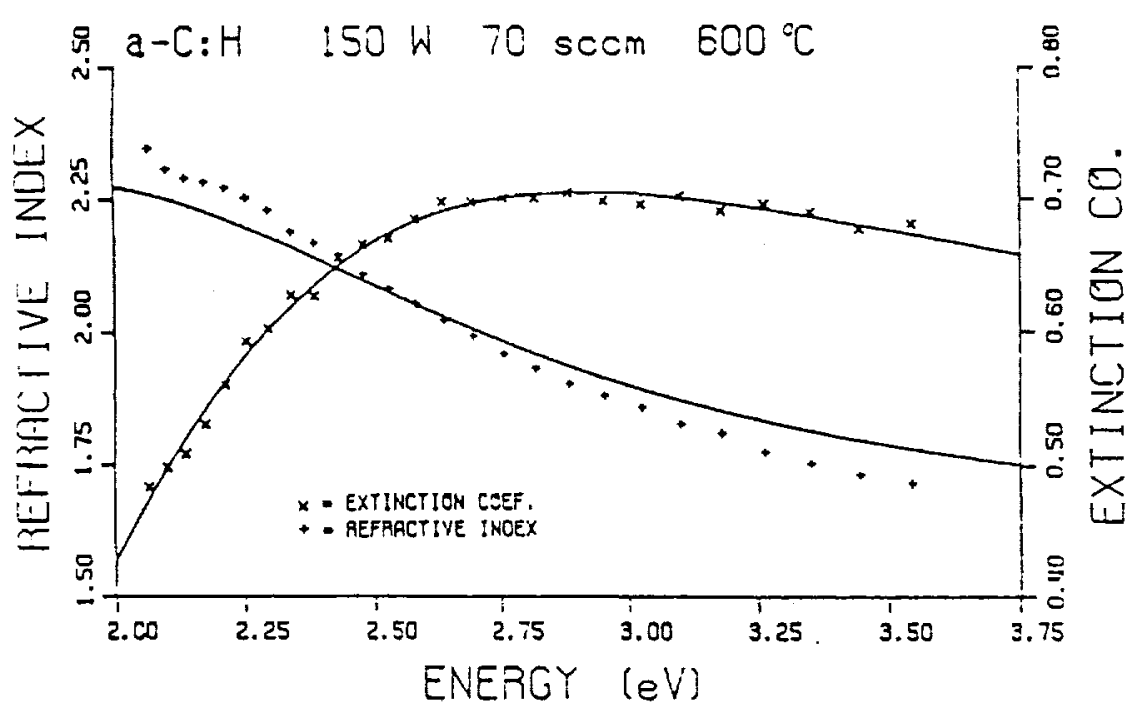

FIGURE 3. REFRACTIVE INDEX AND EXTINCTION COEFFICIENT OF THE I5O $\mathrm{W}, 600^{\circ} \mathrm{C}$ SAMPLE. SOLID LINES ARE DUE TO EQUATIONS (1) AND ( 2 ).

\section{DISCUSSION}

Optical energy bandgaps Eg found using the two Tauc procedures are identical. However, $\varepsilon_{g}$ found using the dispersion relation fits are almost always smaller than the Tauc plots, although the differences are not very large. It is impossible to rule which bandgap is the "correct" one. The dispersion relation functions do take into account, at least qualitatively, the existence of absorption at lower energy than the regular Tauc regime, the Urbach tail. This can be seen by the very small slope of $k(E)$ near Eg, as shown in Fig. 2. However, $\alpha(E)$ is expected to be purely exponential in the Urbach edge [1]. Thus, theoretically, the dispersion relations do not describe this regime. Table I also shows a decrease in Eg with increasing deposition power, (except the $50 \mathrm{~W}$ sample) and with increasing annealing temperature, in agreement with prior results $[4,5]$. The thickness increases with power [16] and decreases with annealing temperature. The quality of the fits shown in Figs. 2 and 3 is quite good. The $n(E)$ fits are the real test of the theory and the figures show a reasonable agreement between experiment and calculation. All other $n(E)$ experimental curves show an equal or better $f i t$ to theory, as they include almost constant $n(E)$ values. In addition, the $k(E)$ fit is definitely better than the Tauc fits.

The value of the A, B,C parameters, as shown in Table II, are essentially constant as function of deposition power, but they change significantly versus annealing temperature. The value of the lifetime $\mathrm{h} / \mathrm{\tau}$, deduced from $\left(4 C-B^{2}\right) / / 2$, has quite an amount of scatter. However, all results are around $h / \tau \cong 2 \mathrm{eV}$. This is a large value, but it is comparable with other amorphous materials [2]. The values of $A$ are in general lower than for other materials [2], denoting a smaller position matrix element $M$ in $a-C: H$. There is an order of magnitude increase in $M$ with heating to $750^{\circ} \mathrm{C}$. It would be interesting to correlate this increase with the changes in the composition and crystallivity of a-C:H. The value of $B$ for the room temperature deposited samples, is equal to the lowest values obtained for other materlals [2], and drops markedly with annealing temperature. The value of $B / 2$ is characteristic of the critical point bandgap [6], denoting a sharp decrease in this bandgap. in parallel with the sharp Eg drop. However, we did not see signs of a peak in $k(E)$ at $E=8 / 2$, as expected for a critical point. 
CONCLUSION

Dispersion relations suggested in [2] are obeyed, although the $n$ (E) function does not have a perfect $f$ it. Values of the garameters for the bandgaps $B$ and $E g$ show reasonable agreement with fauc plots and with prior results. The position matrix element increases with increasing annealing temperature.

\section{REFERENCES}

1. N.F. Mott and E.A. Davis, Electronic Processes in Non-Crystalline Materials, 2nd ed. (Clarendon Press, Oxford, 1979).

2. A.R. Forouhi and I. Bloomer, Phys. Rev. B, 34, 7018 (1986).

3. J. Robertson, Adv. Phys., 35, 317 (1986).

4. S.A. Alterovitz, J.D. Warner, D.C. Liu and J.J. Pouch, J. Electrochem. Soc., 133, 2339 (1986).

5. S.A. Alterovitz, J.J. Pouch and J.D. Warner, in Rapid Thermal Processing of Electronic Materials, edited by S. R. Wilson, R. Powell, and D.E. Davies, (Mater. Res. Soc. Proc. 92. Pittsburgh, PA, 1987) pp. $311-318$.

6. A.R. Forouhi and I. Bloomer, Phys, Rev. B, 38, 1865 (1988).

7. S.A. Aiterovitz, G.H. Bu-Abbud, J.A. Woollam, and D.C. Liu, J. Appl. Phys., 54, 1559 (1983).

8. G.H. Bu-Abbud, S.A. Alterovitz, N.M. Bashara and J.A. Woollam, J. Vac. SCi. Technol., A, 1, 619 (1983).

9. G.H. Bu-Abbud, N.M. Bashara, and J.A. Woollam, Thin Solid Films, 138. $27(1986)$.

10. P.G. Snyder, M.C. Rost, G.H. Bu-Abbud, J.A. Woollam, and S.A. Alterovitz, J. App1. Phys., 60, 3293 (1986).

11. S.A. Alterovitz, J.A. Woollam, and P.G. Snyder, Sol. State Tech., 31 (3), $99(1988)$.

12. J.J. Pouch, J.D. Warner, D.C. Liu and S.A. Alterovitz, Thin Solid Films, 157, 97 (1988).

13. J.A. Woollam Co., Lincoln, NB.

14. D.E. Aspnes and A.A. Studna, Appl. Opt., 14, 220 (1975).

15. R.W. Collins, Appl. Phys. Lett., 52, 2025 (1988).

16. J.J. Pouch, S.A. Alterovitz, J.O. Warner, D.C. Liu, and W.A. Lanford, in Thin Films: The Relationship of Structure to Properties, edited by C.R Aita and K.S. Sreeharsha, (Mater. Res. Soc. Proc. 47, Pittsburgh. $P A, 1985)$ pp. 201-204.

This paper also appears in Mat. Res. Soc. Extended Abstract (EA-19) 